\title{
The role of prefrontal cortex in resolving distractor interference
}

\author{
AMISHI P. JHA, SARA A. FABIAN, and GEOFFREY K. AGUIRRE \\ University of Pennsylvania, Philadelphia, Pennsylvania
}

\begin{abstract}
We investigate the hypothesis that those subregions of the prefrontal cortex (PFC) found to support proactive interference resolution may also support delay-spanning distractor interference resolution. Ten subjects performed delayed-recognition tasks requiring working memory for faces or shoes during functional MRI scanning. During the 15-sec delay interval, task-irrelevant distractors were presented. These distractors were either all faces or all shoes and were thus either congruent or incongruent with the domain of items in the working memory task. Delayed-recognition performance was slower and less accurate during congruent than during incongruent trials. Our fMRI analyses revealed significant delay interval activity for face and shoe working memory tasks within both dorsal and ventral PFC. However, only ventral PFC activity was modulated by distractor category, with greater activity for congruent than for incongruent trials. Importantly, this congruency effect was only present for correct trials. In addition to PFC, activity within the fusiform face area was investigated. During face distraction, activity was greater for face relative to shoe working memory. As in ventrolateral PFC, this congruency effect was only present for correct trials. These results suggest that the ventrolateral PFC and fusiform face area may work together to support delay-spanning interference resolution.
\end{abstract}

Patricia Goldman-Rakic was a scientific pioneer. She ventured into the uncharted territory of investigating prefrontal cortex (PFC) and successfully mobilized an entire generation of working memory researchers to follow her. Her influential finding that specialized cells within PFC fire in a sustained fashion during working memory tasks (Funahashi, Bruce, \& Goldman-Rakic, 1989; GoldmanRakic, 1987) has served as a signpost for all subsequent inquiry regarding the functional organization of PFC and the neural bases of working memory. She proposed a dorsal/ventral segregation of PFC based on the domain of information maintained in working memory, with dorsal subregions for spatial working memory and ventral subregions for object working memory (Rao, Williams, \& Goldman-Rakic, 1999; Wilson, Ó Scalaidhe, \& GoldmanRakic, 1993). In addition, she proposed that each of these domain-specific PFC modules was interconnected with domain-specific posterior regions (Cavada \& GoldmanRakic, 1989). She argued that the coordinated sustained firing of these regions $i$ s the neural instantiation of working memory maintenance operations (Chafee \& GoldmanRakic, 1998, 2000).

Goldman-Rakic's model emphasized working memory maintenance operations and their "domain-differentiated"

We thank Sharon Thompson-Schill for helpful discussions and Elaine Wencil and Jason Krompinger for assistance in data collection and analysis. This research was supported by a grant from the University of Pennsylvania Research Foundation. Correspondence should be addressed to A. P. Jha, Center for Cognitive Neuroscience, University of Pennsylvania, 3401 Walnut St., Suite 302C, Philadelphia, PA 19104 (email: apjha@psych.upenn.edu). neural organization, but other models propose that maintenance is only one of several processes critical for successful working memory performance (Fuster, 1997; Luria \& Homskaya, 1970). These models suggest a processdifferentiated organization of PFC in which maintenance, manipulation, response inhibition, interference resolution, and task-switching are all subserved by distinct subregions of PFC (see Fuster, 1997, for a review).

One prominent process-differentiated model posits that maintenance is subserved by dorsolateral regions of PFC, and inhibitory processes such as distractor management, response inhibition, and interference resolution are subserved by ventrolateral PFC (Luria \& Homskaya, 1970). This model was motivated by the finding that PFC lesions result in performance impairments specifically when patients must discriminate between relevant and irrelevant information. Individuals with PFC lesions have been reported to be more vulnerable to distraction (Chao \& Knight, 1995; Knight, Hillyard, Woods, \& Neville, 1981; Malmo, 1942), unable to inhibit prepotent responses (Luria \& Homskaya, 1970), and unable to overcome interference (Thompson-Schill et al., 2002). These processes have been subsumed under the heading of behavioral inhibition, but recent neuroimaging studies suggest that ventrolateral PFC may be more generally involved in selection between competing alternatives than in inhibition of irrelevant information per se (Sakai, Rowe, \& Passingham, 2002; Thompson-Schill, 2003; ThompsonSchill, D’Esposito, Aguirre, \& Farah, 1997; Tippett, Gendall, Farah, \& Thompson-Schill, 2004).

These neuroimaging studies were done in the context of delayed-recognition paradigms in which interference 
was manipulated across trials. Trials began with the presentation of the memory array (S1), which was followed by a brief delay interval, and ended with a probe item (S2). The subject's task was to determine if S2 was a member of the S1 memory array on that trial. The critical manipulation required the subjects to correctly respond "no" when S2 had been a member of S1 from a previous trial (recent-negative probe) but was not a member of $\mathrm{S} 1$ on the current trial. Behaviorally, the subjects were slower and less accurate in correctly responding to these recentnegative probe trials relative to other trial types (Goggin \& Wickens, 1971; Hawkins, Pardo, \& Cox, 1972; Marcer, 1972; May, Hasher, \& Kane, 1999; McIntyre, Stojak, \& Mostoway, 1973).

Numerous studies have now confirmed that ventrolateral PFC is more active in these recent-negative trials than in other trial types (Bunge, Ochsner, Desmond, Glover, \& Gabrieli, 2001; D'Esposito, Postle, Jonides, \& Smith, 1999; Jonides, Smith, Marshuetz, Koeppe, \& Reuter-Lorenz, 1998). Thompson-Schill and colleagues (Thompson-Schill, 2003; Thompson-Schill et al., 1997; Tippett et al., 2004) propose that selection processes are summoned by the sense of familiarity that accompanies presentation of recent-negative trials. Selection then permits elaborated evaluation of items to determine their taskrelevant or -irrelevant status. This proposal predicts that ventrolateral PFC activity may be greater when certainty regarding the item's task-relevance is low and the need for selection is high, relative to when certainty is high and the need for selection is low. Indeed, tasks manipulating semantic (Kan \& Thompson-Schill, 2004; ThompsonSchill, 2003; Tippett et al., 2004) and mnemonic selection (Nelson, Reuter-Lorenz, Sylvester, Jonides, \& Smith, 2003; Thompson-Schill et al., 2002) but not response selection (Milham et al., 2001) report greater ventrolateral PFC activity when selection demands are high versus low. Thus, together these results suggest that ventrolateral PFC may support representational selection processes to resolve interference.

Representational selection is akin to perceptual selection processes described in models of selective attention (for a review, see Kan \& Thompson-Schill in this issue). Researchers have proposed that attentional selection during perception operates via prefrontal biasing of posterior perceptual regions. This biasing favors processing of task-relevant items and inhibits processing of simultaneously presented task-irrelevant items (Desimone \& Duncan, 1995; Kastner, Pinsk, De Weerd, Desimone, \& Ungerleider, 1999). Recently, attentional selection has been suggested as the mechanism by which information is actively maintained in working memory (Awh, Jonides, \& Reuter-Lorenz, 1998; Awh et al., 1999; Barnes, Nelson, \& Reuter-Lorenz, 2001; Corbetta, Kincade, \& Shulman, 2002; Jha, 2002; LaBar, Gitelman, Parrish, \& Mesulam, 1999). Selection mechanisms, in the context of working memory, may bias both prefrontal and posterior perceptual activity in favor of memoranda during the entire period of active maintenance (Awh et al., 1999; Jha, 2002).
Although some models of PFC suggest a neuroanatomic segregation and functional dissociation between maintenance and interference processes (Fuster, 1995; Luria, 1973; Luria \& Homskaya, 1970), some models of working memory do not support this theoretical distinction (Desimone, Chelazzi, Miller, \& Duncan, 1995; Kimberg \& Farah, 1993; Miller \& Cohen, 2001). According to Kimberg and Farah, for example, both maintenance and interference resolution are emergent properties of a functional circuit that weights processing in favor of relevant information. One challenge for empirical tests investigating the neural bases of maintenance and interference processes is that activity profiles must be compared across distinct nonoverlapping trial components during different experimental manipulations. Whereas maintenance activity is examined during the delay interval and typically involves load manipulations during delayed-recognition tasks (Jha \& McCarthy, 2000; Leung, Gore, \& GoldmanRakic, 2002; Ranganath, DeGutis, \& D’Esposito, 2004; Rypma, Prabhakaran, Desmond, Glover, \& Gabrieli, 1999), interference-related activity is generally examined during S2-evoked activity and compares recentnegative trials with other trial types (D'Esposito, Postle, Jonides, \& Smith, 1999).

In the present study, we manipulated interference during the delay interval, when maintenance processes were presumably ongoing. The subjects performed working memory delayed-recognition tasks for faces or shoes. During the 15-sec delay interval, task-irrelevant distractors were presented. These distractors were either congruent (e.g., face distractors during face working memory) or incongruent (e.g., shoe distractors during face working memory) with the working memory domain, but never appeared as S1 or S2 stimuli. During these trials, we examined fMRI activity within dorsal and ventrolateral PFC, as well as the fusiform face area (FFA).

The attentional rehearsal hypothesis suggests that activity within prefrontal and posterior perceptual regions, such as the FFA, will be biased in favor of maintained stimuli during the entire delay interval. With this maintenancerelated bias in place, it is possible that perceptual processing of task-irrelevant distractors may be facilitated when there is a high degree of overlap between their features and the features of items being maintained in working memory (e.g., congruent distractors). We propose that under these conditions, selection mechanisms may be invoked during the delay interval to classify distractors appropriately as irrelevant, and conceivably to inhibit their processing. Our three hypotheses are as follows: First, if ventrolateral PFC subserves representational selection processes needed for successful maintenance, delay-interval activity within ventrolateral PFC will be greater during congruent than during incongruent distraction. Second, if ventrolateral PFC biases posterior-perceptual processing, activity within FFA during presentation of face distractors will also be modulated by congruency with the working memory task. Third, if selection processes are needed to segregate relevant and irrelevant information effectively in the service of 
successful working memory performance, distractor congruency effects will be more pronounced during correct than during incorrect working memory performance.

\section{METHOD}

\section{Subjects}

Ten ( 5 female) healthy, right-handed volunteers (18-24 years of age; mean 21.1 years) participated in this study. Two subjects were removed from analyses due to data acquisition software errors. The University of Pennsylvania Institutional Review Board approved this study, and each subject provided informed consent and received payment for participating.

\section{Behavioral Task}

The subjects were scanned during performance of a delayedrecognition task (Figure 1). The memory array (S1) in each trial and consisted of two images, one presented to the right and another to the left of a central fixation cross for $3 \mathrm{sec}$. S1 offset was followed by a 15 -sec delay interval. At the end of the delay, S2 was presented in the center of the display for $3 \mathrm{sec}$. S2 was a single image from the S1array on half of the trials and consisted of a novel image on the remaining trials. The subject's task was to determine whether S2 matched either of the items in S1 and to press a button indicating a "match" or "nonmatch" response. The intertrial interval (ITI) was $16.5 \mathrm{sec}$. Half of the trials required working memory for faces, and the other half required working memory for shoes. These trial types were randomly intermixed throughout the experiment. A total of 128 trials were presented over eight experimental runs.

For all stimuli, great care was taken to ensure that stimulus features were difficult to verbally encode. A prescreening of stimuli was done with 10 other subjects to ensure that our face and shoe image databases contained no images with distinguishing features for faces (prominent eyebrows, facial hair, affective expressions) or shoes (brand names, distinguishing shoe style) (Wencil, Rauch, Scheinker, \& Jha, 2003).

During face working memory trials, S1 and S2 face images were surrounded by ovals to mask hair and clothing. During shoe working memory trials, $\mathrm{S} 1$ and $\mathrm{S} 2$ consisted of shoe images. On all trials, taskirrelevant distractors, consisting of faces or shoes, were presented during the delay interval. The subjects were told simply to hold their eyes at fixation throughout the delay and to disregard these distractors. Each distractor array was presented for $1 \mathrm{sec}$ and consisted of six identical images presented in an imaginary grid above and below a central fixation cross. Twelve distractor arrays were presented during the final $12 \mathrm{sec}$ of each delay interval. Each distractor array was presented only once during the entire experiment, and the picture never appeared as on S1 or on S2. All distractor arrays presented during a single trial were always from the same stimulus category (e.g., all faces or all shoes). Distractors were faces on half of all trials and shoes on the other half, and these trial types were randomly intermixed.

In addition to the delayed-recognition task, the subjects performed a face localizer task to identify face-sensitive regions within occipitotemporal cortex (Epstein \& Kanwisher, 1998; Kanwisher, McDermott, \& Chun, 1997). During two experimental runs, faces, objects, and scrambled objects were presented in 18-sec blocks. Each stimulus was presented for $500 \mathrm{msec}$ followed by a $500-\mathrm{msec}$ interstimulus interval.

All stimuli were displayed using a Dell Inspiron 4100 laptop and were projected with an Epson LCD projector onto a screen placed at the subject's head and viewed through an angled mirror.

\section{MRI Acquisition and Processing}

Anatomical and functional data were acquired using a $3.0 \mathrm{~T}$ Siemens Trio scanner with a standard head coil. High-resolution
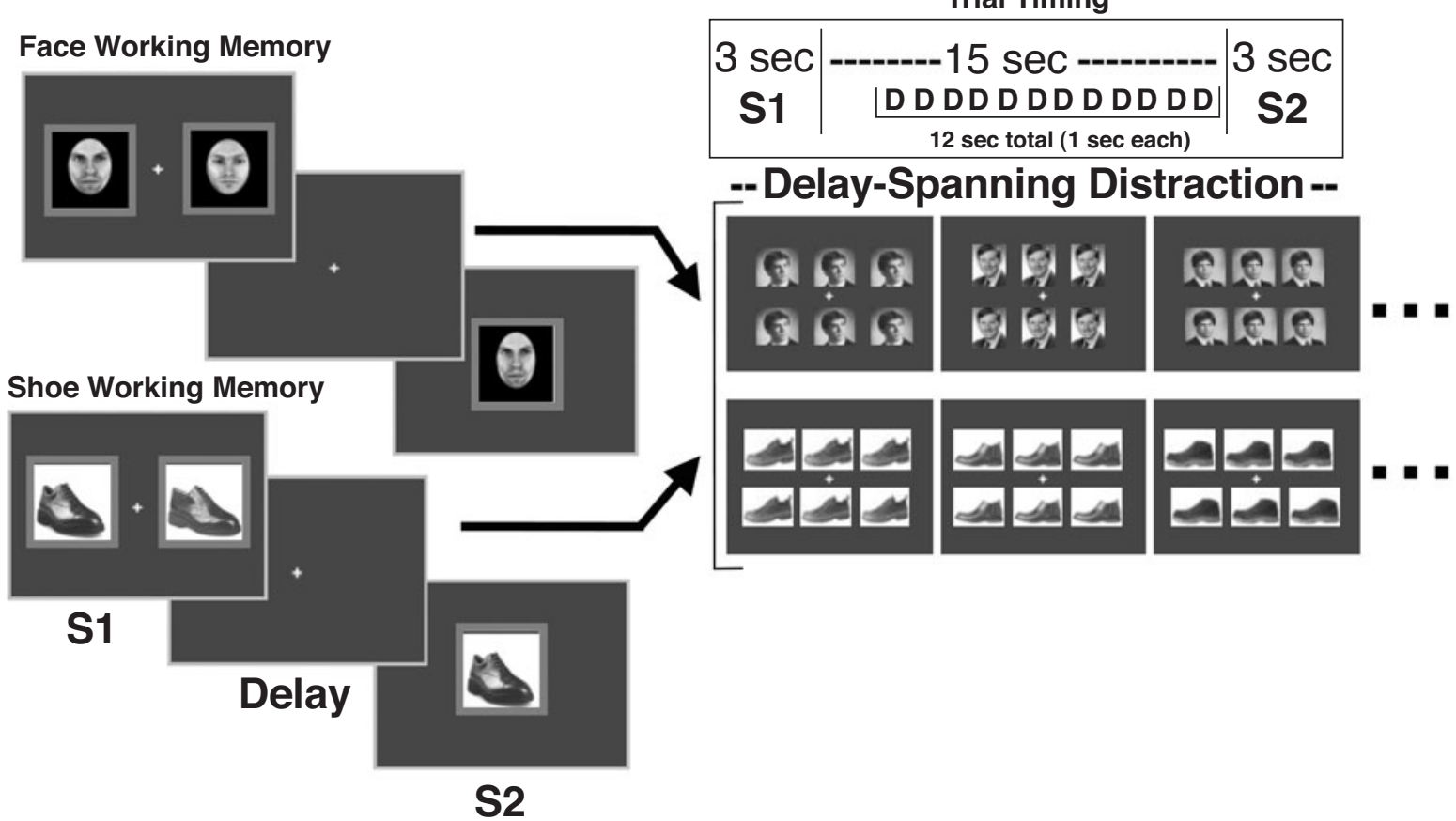

Figure 1. Schematic of behavioral paradigm and task design. Each trial began with the presentation of the memory set (S1), followed by a 15-sec delay interval, and ended with the test item (S2), to which a response was executed. Two types of distractors were presented during the delay. On half of the trials, face distractors were presented, and on the remaining trials, shoe distractors were presented instead. 
axial T1-weighted images were obtained from each subject prior to functional imaging. A total of 160 axial slices consisting of nearisotropic voxels $(0.9766 \times 0.9766 \times 1 \mathrm{~mm})$ were acquired $(192 \times$ $256 \times 160$ voxel matrix, flip angle $=15^{\circ} ; \mathrm{FOV}=25, \mathrm{TE}=3 \mathrm{msec}$, $\mathrm{TR}=1,620 \mathrm{msec}, \mathrm{BW}=150, \mathrm{TI}=950 \mathrm{msec}$, field strength $=$ 2.89362)

Blood oxygenation level dependent (BOLD) echo-planar functional images ( $\mathrm{TR}=3,000 \mathrm{msec}, \mathrm{TE}=30 \mathrm{msec}$ ) were acquired axially for 10 experimental runs. Each brain volume consisted of 44 neighboring 3-mm slices, with an in-plane resolution of $64 \times 64$, yielding a voxel size of $3 \times 3 \times 3 \mathrm{~mm}(\mathrm{FOV}=25$, TI $=-1.99$, $\mathrm{BW}=3,256$, field strength $=2.89362$ ) for full-brain coverage Eighty-four brain volumes were acquired during two runs of the localizer task. A further 204 brain volumes were acquired for each of the 8 delayed-recognition task runs.

\section{Behavioral Data Analysis}

Behavioral data were analyzed independently of functional data. Response time (RT) and percentage correct measures were entered into separate analysis of variance (ANOVA) tests to determine averages and statistically significant differences as a function of condition.

\section{Imaging Data Analysis}

The VoxBo software package (www.voxbo.org) was used for all fMRI analyses. Standard data preprocessing included slice-time correction, six-parameter motion correction (Friston et al., 1995), and conversion of orientation to the coronal plane. Spatial smoothing and spatial normalization were not conducted, as hypotheses were to be tested in anatomically and functionally defined regions of interest (ROIs).

The general linear model (GLM) was used to statistically characterize fMRI signal changes observed in the time-series analyses as a series of amplitude-scaled and time-shifted covariates. This method has been extensively used and is thoroughly described elsewhere (Postle, Zarahn, \& D'Esposito, 2000; Zarahn, Aguirre, \& D'Esposito, 1997a, 1999). Briefly, changes in BOLD signal were modeled as independent regressors for each stage of each condition of the working memory task (e.g., face working memory: S1-evoked activity). A single regressor time-locked to stimulus onset was used to model both $\mathrm{S} 1$-evoked activity ( $0-3 \mathrm{sec}$ following $\mathrm{S} 1$ onset) and S2-evoked activity (18-21 sec following S1 onset). The delay interval was modeled by three regressors at $7 \mathrm{sec}, 12 \mathrm{sec}$, and $15 \mathrm{sec}$ following S1 onset. Each regressor was convolved with a canonical hemodynamic response function (HRF). Data were proportionally normalized to account for differences in mean signal values and to correct for linear signal drift within scanning runs. Additional nuisance covariates were included to model the intercept and trialspecific baseline shifts. The convolution matrix included a time domain representation of the $1 / f$ power structure (Zarahn et al., 1997a, 1997b) and a filter to remove frequencies above and below the prominent task frequencies. For each subject, beta values (regression weights) yielded by the GLM for the S1, delay, and S2 time periods were extracted for each covariate and averaged within each functionally defined ROI. The beta values served as dependent measures for across-subjects random-effects analyses. Because our hypotheses concerned activity changes specific to the delay interval, analyses centered on the covariates modeling this task period.

\section{ROI Analyses}

Activity during working memory trials was examined within three functionally defined ROIs - dorsolateral PFC, ventrolateral $\mathrm{PFC}$, and FFA. All regions were first defined by anatomical boundaries. Anatomical ROIs within dorsolateral and ventrolateral PFC were identified on each subject's high-resolution coronal anatomical images by experienced technicians. ROIs were drawn on left and right middle frontal gyri (MFG) and inferior frontal gyri (IFG), to correspond to the dorso- and ventrolateral $\mathrm{PFC}$ regions, respectively. These regions were drawn over three contiguous slices to cre- ate three discrete slice bands (Figure 2: Regions 1, 2, and 3). ROIs were indexed by the distance in millimeters from each subject's anterior commissure (AC) so that summary activation waveforms by slice region could be created across subjects for each region. These prefrontal ROIs ranged from 9-36 mm anterior to the AC. Slice bands were selected a priori based on regions reported to be activated in previous delayed-recognition and proactive interference studies (Bunge et al., 2001; D'Esposito, Postle, Ballard, \& Lease, 1999; D'Esposito, Postle, Jonides, \& Smith, 1999; Leung et al., 2002; Nelson et al., 2003; Thompson-Schill et al., 2002). Next, an ROI was defined as all voxels within these regions that exhibited statistically significant task-related activity $(p<.05$, uncorrected) during any phase of the delayed-recognition task. This method of identifying functional-anatomical ROIs has been recently used to examine prefrontal and posterior perceptual activity (Ranganath et al., 2004).

Anatomical ROIs for the FFA first required locating the fusiform gyrus anatomically. Next, the FFA was defined as all voxels within the left and right fusiform gyrus showing statistically significant activation ( $p<.05$, uncorrected) during the localizer task in a contrast of faces versus objects. An important aspect of the statistical contrasts used to functionally define ROIs was that they were orthogonal to, and therefore did not bias, the subsequent statistical contrasts conducted within these ROIs.

\section{fMRI Planned Contrasts}

BOLD responses during the working memory task were separately analyzed for functional-anatomical ROIs within right and left dorso- and ventrolateral PFC and FFA separately for correct and incorrect working memory trials. We modeled our data to assess independent activity associated with $\mathrm{S} 1, \mathrm{~S} 2$, and the delay interval, and used a long delay interval duration $(15 \mathrm{sec})$ to ensure that our contrasts of interest regarding delay-spanning distraction were uncontaminated by S1- or S2-evoked activity.

\section{Anatomically Defined ROls Within Dorsolateral and Ventrolateral PFC}

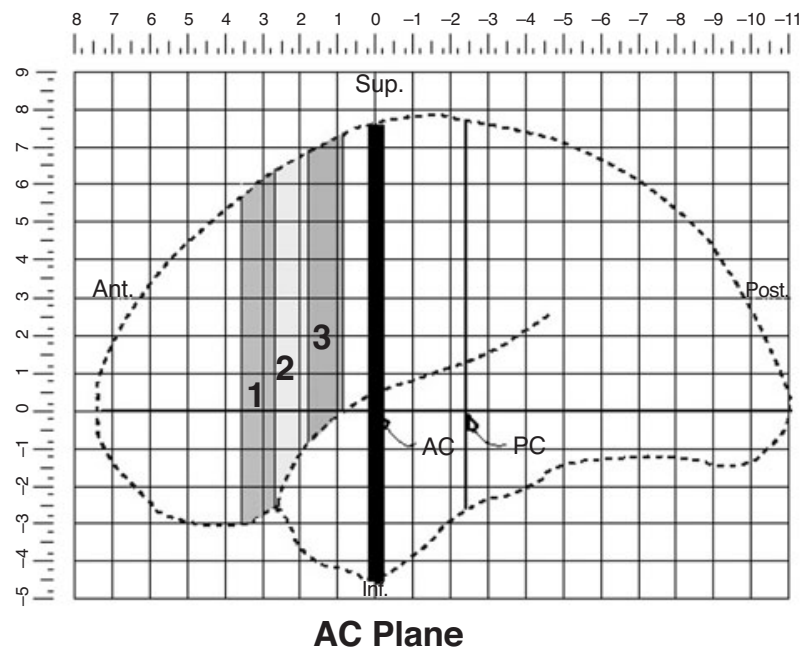

Figure 2. ROI determination. ROIs within dorso- and ventrolateral prefrontal cortex were drawn on a slice-by-slice, subjectby-subject basis. All regions were indexed relative to the distance from the anterior commissure (AC), which allowed for acrosssubjects comparisons without spatial normalization. Three slice bands within the middle and inferior frontal gyrus were investigated. 
Prefrontal cortex. Our overall goal was to determine which regions of PFC were sensitive to the category of delay-spanning distraction. We hypothesized that activity within ventrolateral PFC, which has previously been reported to subserve representational selection mechanisms during proactive interference paradigms (D'Esposito, Postle, Jonides, \& Smith, 1999; Milham et al., 2001; Nelson et al., 2003; Thompson-Schill et al., 2002), may also subserve delayspanning distractor interference resolution. Specifically, we conjectured that selection mechanisms would enable appropriate segregation of relevant information (i.e., active contents of working memory) from irrelevant information (i.e., task-irrelevant distractors). Furthermore, selection demands corresponding to ventrolateral PFC activity were predicted to be greater during congruent than during incongruent distraction. Thus, our contrast of interest compared delay interval activity for congruent and incongruent distraction, with face and shoe working memory trials analyzed separately.

Congruency was determined by holding the working memory domain constant (e.g., face working memory) and varying the type of distractor that was presented (e.g., congruent face distractors or incongruent shoe distractors). Each subject contributed a normalized effect score that indexed his or her distractor interference effect. Separate analyses were conducted for each of the PFC regions (dorso- and ventrolateral PFC). Within each region, the effect scores were first entered into three-way ANOVAs to determine whether the effect sizes significantly differed across working memory tasks (face vs. shoe working memory), slice bands $(1,2$, or 3 ), or hemisphere (right vs. left). This analysis allowed us to determine whether distinct subregions were maximally activated for different working memory tasks. Once peak-activity regions were identified, effect scores for those regions were entered into one-sample $t$ tests to determine whether they differed significantly from zero.

Fusiform face area. We reasoned that greater selection demands would be needed to segregate the relevant contents of working memory from task-irrelevant distraction during congruent than during incongruent delay-spanning distraction. As reviewed above, we hypothesized that ventrolateral PFC is the "source" controlling selection operations and predicted that its activity would be greater for congruent than for incongruent trials. In addition, we proposed that the FFA is the recipient "site" of prefrontal control.

The analysis approach outlined above for the PFC holds constant the working memory task domain and compares activity during face versus shoe distraction. A similar analysis approach for the FFA would likely yield greater activity for face than for shoe distractors, regardless of their congruency with the working memory task, because faces are the preferred stimulus class for the FFA.

Since our goal was to determine whether category congruency, rather than domain of the stimulus, modulates activity, analyses of the FFA held constant the domain of the distractors while defining them as congruent or incongruent, according to the domain of the working memory task for a particular trial. The contrast of interest compared delay activity during face versus shoe working memory separately for face and shoe distractors. We predicted very little overall activity in the shoe distractor conditions because FFA is not robustly activated by complex nonface objects (McCarthy, Puce, Gore, \& Allison, 1997). For face distractors, however, we expected that activity would be robust during both working memory tasks. Furthermore, we predicted differential activity for face distractors during face and shoe working memory.

The effect scores for face versus shoe working memory were first entered into one-way ANOVAs to determine whether effect sizes significantly differed across hemispheres (right vs. left) for face and shoe distractors. Next, effect scores from the most active hemisphere were entered into a one-sample $t$ test to determine whether they differed significantly from zero.

\section{RESULTS}

\section{Behavioral Results}

Behavioral results are shown in Figure 3. Separate two-way ANOVAs were conducted for RT and accuracy results to investigate performance differences as a function of congruency (congruent vs. incongruent) and working memory domain (faces vs. shoes). RTs were significantly slower on congruent relative to incongruent distractor trials $[F(1,6)=8.55, p<.03]$. Furthermore, there was a near-significant effect of working memory domain, with slower RTs during shoe than during face working memory $[F(1,6)=5.4, p<.06$; mean RTs - for faces $=1,111 \mathrm{msec}$, for shoes $=1,190 \mathrm{msec}]$. There was also a near-significant interaction of congruency and working memory domain $[F(1,6)=4.1, p<.09]$. Accuracy scores were lower for incongruent than for congruent trials, but this effect did not reach statistical significance $[F(1,6)=4.7, p<.08]$. In addition, accuracy was significantly lower for shoe than for face working memory $[F(1,6)=8.57, p<.03]$, but there was no significant interaction of congruency and working memory domain $[F(1,6)=.04, p>.89]$.

\section{Imaging Results}

Delay-spanning distractor interference effect scores were obtained to contrast congruent and incongruent

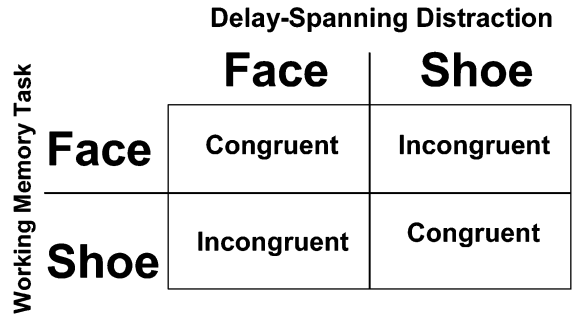

Figure 3. (Left) Task conditions. There were four task conditions defined by the category congruency between working memory domain and distractor domain. All trial types were randomly intermixed and equally probable. (Right) Behavioral performance. Graphs show mean response times and accuracy during working memory task performance as a function of distractor congruency. Responses were slower and less accurate during congruent trials, suggesting that distractors were more behaviorally intrusive during these trials relative to incongruent distractor presentation. 
distraction for correct trials. These scores were entered into separate three-way ANOVAs for the dorso- and ventrolateral PFC ROIs. For the dorsolateral PFC, there was no main effect of working memory task domain (face vs. shoe), slice band $(1,2$, or 3 ), or hemisphere (left vs. right), nor were there any interactions between these terms $(p>.3)$. The scores for the region as a whole, collapsed across slices and hemispheres were entered into one-sample $t$ tests to determine whether the magnitude of the effect differed significantly from zero, and this result was also not significant ( $p>.7$ ). It is important to note that although the effect scores for the contrast of interest were not significant for the dorsolateral PFC, robust delay interval activity was observed within this region (as might be expected given the manner in which the regions were defined), and the overall level of activity during the delay interval did not differ from the overall level of activity observed within ventrolateral PFC $(p>.23)$.

For the ventrolateral PFC, there was no main effect of working memory domain $(p>.26)$. A significant main effect of hemisphere was observed $[F(1,7)=9.26, p<$ $.01]$, with greater effect scores for the left than for the right hemisphere. There was also a main effect of slice band $[F(2,14)=6.73, p<.02]$. A secondary contrast revealed that Slice Bands 2 and 3 were significantly more active than Slice Band $1[F(1,7)=18.53, p<.008]$ but did not significantly differ from each other $(p>.2)$. There were no significant interactions between any of the factors $(p>.16)$. Thus, the region of the ventrolateral PFC most significantly modulated by delay-spanning distractor congruency was the posterior extent of the left IFG (LIFG).

The effect scores from the posterior LIFG for all subjects were then entered into a one-sample $t$ test to determine whether the effects significantly differed from zero (see Figure 4). Effect scores for both face $[t(7)=2.6$, $p<.04, M=0.8]$ and shoe $[t(7)=2.58, p<.04, M=$ $0.38]$ working memory tasks significantly differed from zero. The overall magnitude of the effect, however, was greater in the face than in the shoe working memory task. This pattern is consistent with the magnitude of the behavioral interference effect. In the right panel of Figure 4, we show the time-series data for the posterior LIFG. Although our statistical analyses emphasized the delay interval, it is important to note that only the delay activity differed across the distractor conditions. Examination of correct trials, as reviewed above, revealed significant distractor congruency effects within LIFG, but no significant congruency effect was observed for incorrect trials. A direct comparison of effect scores between correct and incorrect trials revealed that they significantly differed from each other within this region $[t(7)=2.77, p<.03]$.

Thus, effect scores within LIFG were significantly greater during correct than during incorrect trials. Although there were far fewer incorrect than correct trials, it is important to note that statistical power for both trial types was equivalent, because both correct and incorrect trials were modeled with a common error term within our statistical model (see Zarahn et al., 1997a, 1997b). Nonetheless, we sought to directly test the hypothesis that correct performance may necessitate LIFG activity. That is, if ac-

\section{Left Ventrolateral PFC}
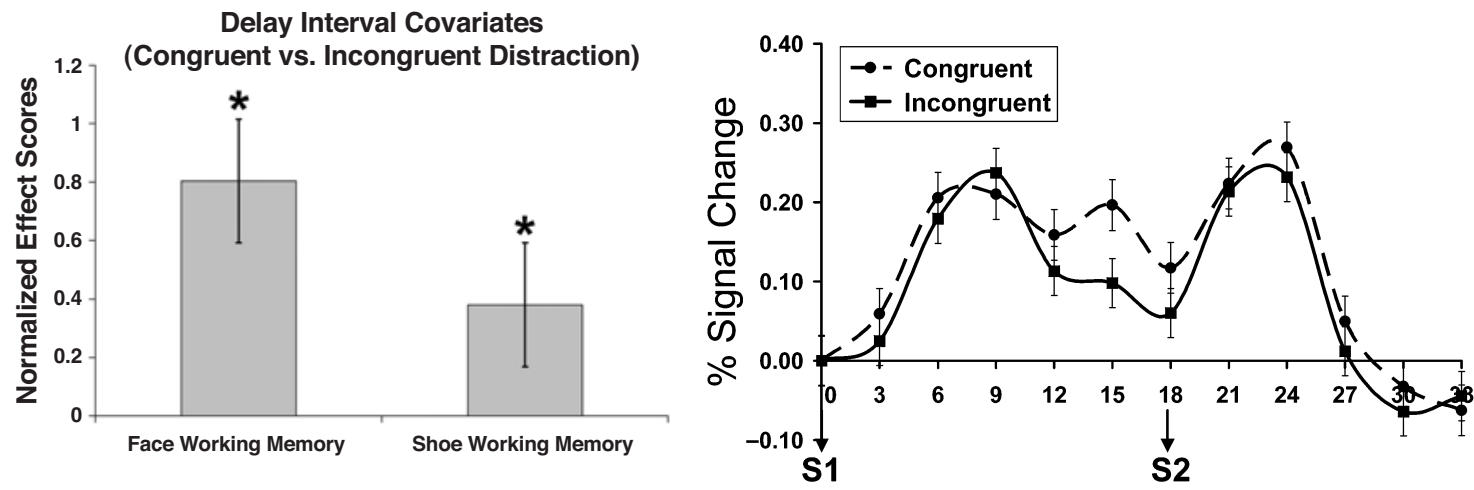

Figure 4. (Left) Ventrolateral PFC activity by working memory domain. The bar graph shows normalized parameter estimates for face and shoe working memory collapsed across Slice Bands 2 and 3 within the left ventrolateral PFC for the contrast between congruent and incongruent delay-spanning distraction. Error bars denote standard errors of the mean across subjects. These results show that left ventrolateral PFC activity was significantly greater during the delay interval for both face and shoe working memory tasks during congruent trials. However, the magnitude of the distractor congruency effect was more robust for faces relative to shoes. (Right) Ventrolateral PFC time series. The time series presented in this graph is the average percentage change in $\mathrm{fMRI}$ signal within left ventrolateral PFC over the course of delayed-recognition trials (collapsed across face and shoe working memory). Time series are shown for congruent (dotted line) and incongruent (solid line) trial types. Error bars denote standard errors of the mean across subjects. These results graphically depict the significant distractor interference effects observed specifically during the delay interval of these delayed-recognition trials. 
tivity within LIFG is related to performance (i.e., success or failure on particular trials), then IFG effect scores should be variable during correct performance across subjects. Perhaps subjects who perform well do so because of efficient engagement of selection mechanisms subserved by LIFG. We measured the correlation between each subject's behavioral performance and his or her LIFG effect score. The correlation was highly significant at $r=.73$ $(p<.005)$. Thus, even when error trials were not considered, there still appeared to be a brain-behavioral relationship according to which successful performance corresponded to robust LIFG activity.

\section{Fusiform Face Area}

An ANOVA with a single factor (hemisphere of FFA ROI: left vs. right) was performed on effect scores for face versus shoe working memory separately for face and shoe distractors. There was a significant main effect of hemisphere for faces $[F(1,7)=5.96, p<.05]$ but not for shoes $(p>.39)$. Effect scores were greater in the right than in the left FFA for faces. The effect scores for face distractors were positive, indicating that delay-spanning activity was greater during face relative to shoe working memory trials. In addition, these scores were significantly greater than zero for face $[t(7)=2.6, p<.04$; $M=1.2]$ but not for shoe distraction $(p>.16 ; M=0.12)$. Thus, within FFA, the neural responses to faces were greater when these distractors were presented in the context of face rather than shoe working memory (see Figure 5, top panel).

As reviewed above, effect scores for the contrast of interest for the FFA (face vs. shoe working memory) reached

\section{FUSIFORM FACE AREA}

\section{Face vs. Shoe Working Memory}

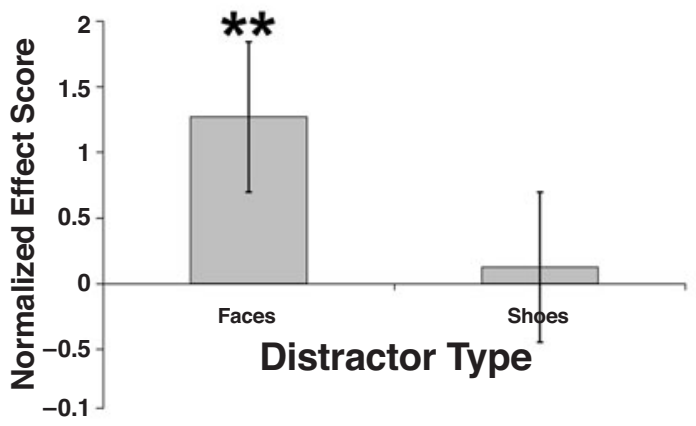

\section{Correct Trials}

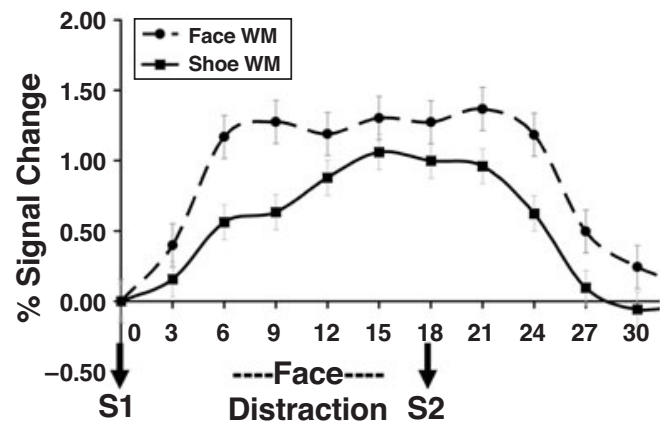

Incorrect Trials

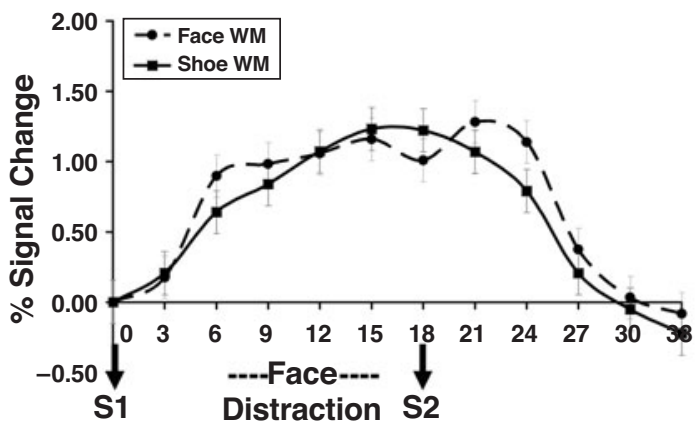

Figure 5. (Top) FFA activity for face and shoe distractors. The bar graph shows normalized parameter estimates for face and shoe distractors for a contrast between delay interval activity during face versus shoe working memory. Error bars denote standard errors of the mean across subjects. Right FFA activity was significantly greater during the delay interval for face relative to shoe working memory tasks only for faces. (Bottom left) FFA time series for correct face distraction trials. The time series presented in this graph is the average percentage change in fMRI signal for correct trials within right FFA over the course of delayed-recognition trials for face (dotted) and shoe (solid) working memory during face distraction. Error bars denote standard errors of the mean across subjects. These results depict the significantly greater activity for face rather than shoe working memory during the delay interval. Greater S1- and S2-evoked activity was also observed for face working memory, reflecting FFA's preference for processing faces during S1 and S2 across working memory tasks. Importantly, when sensoryperceptual content was held constant during the delay interval (e.g., all face distractors), activity still remained greater for face than for shoe working memory within the FFA. (Bottom right) FFA time series for incorrect face distraction trials. When performance was incorrect, although S1- and S2-evoked activity still remained greater during face versus shoe working memory trials, activity during the delay interval did not significantly differ between the two working memory tasks. 
statistical significance only for face distractors and only when performance was correct. In contrast, effect scores did not differ from zero for incorrect trials. The time series during presentation of face distractors is shown in Figure 5 for correct (bottom left) and incorrect (bottom right) trials. The most striking difference between these two graphs is the absence of a congruency-related difference in activity during incorrect trials. A direct comparison of effect scores between correct and incorrect trials revealed that they differed significantly from each other within this region $[t(7)=4.7, p<.003]$.

Below, we discuss possible processes reflected by the congruency-related modulation observed within both ventrolateral PFC and FFA.

\section{DISCUSSION}

Attentional selection during perception tasks involves prefrontal biasing of posterior perceptual regions (Giesbrecht \& Mangun, 2002; Hopfinger, Buonocore, \& Mangun, 2000; Mangun, Buonocore, Girelli, \& Jha, 1998). This biasing leads to enhanced perceptual processing of task-relevant items and relative inhibition of simultaneously presented task-irrelevant stimuli (Desimone \& Duncan, 1995; Kastner et al., 1999). In the present study, we examined the role of selection in working memory. We hypothesized that during working memory tasks, selection processes would be invoked to segregate maintenance-related activity from activity evoked by presentation of delayspanning distractors. Specifically, we predicted that there would be greater need for selection when feature overlap between items maintained in working memory and those presented as distractors was high rather than low. We manipulated feature overlap by the category congruency of memory and distractor items. Greater activity within left ventrolateral PFC was observed when delay-spanning distractors were congruent with the working memory task domain. A similar activity pattern was observed within FFA. In this region, delay-spanning neural activity evoked by face distractors was greater during face rather than shoe working memory trials. Importantly, whereas congruency effects within both regions were robust during correct trials, these effects were absent for incorrect trials. Thus, these results are consistent with the view that successful working memory performance relies on ventrolateral PFC and FFA to resolve distractor interference during delay-spanning distraction.

Although our results demonstrate a high degree of correspondence in activity profiles between ventrolateral PFC and posterior perceptual regions, we did not directly assess the functional connectivity between these regions. An elegant study by Gazzaley and colleagues, in the present issue, examines functional connectivity in the context of face working memory tasks. They report that activity within FFA is significantly correlated with activity within dorso- and ventrolateral PFC, among other regions. Although numerous studies conclude that prefrontal and posterior perceptual regions are both crit- ical for working memory (Chafee \& Goldman-Rakic, 1998, 2000; Constantinidis \& Steinmetz, 1996; Miller, Erickson, \& Desimone, 1996; Miller, Li, \& Desimone, 1991), the precise functions subserved by these regions are under active debate.

One prominent view is that the $\mathrm{PFC}$ and posterior perceptual regions support working memory maintenance processes. Several studies report that the magnitude of delay interval activity within PFC, as well as functional connectivity between PFC and posterior perceptual regions, correlates with behavioral performance. Delay activity was found to be robust on correct trials and weak or absent on incorrect trials within dorso- and ventrolateral PFC (Funahashi et al., 1989; Fuster, 1973; Pessoa, Kastner, \& Ungerleider, 2003; Sakai et al., 2002). This performance-related modulation of delay activity has been proposed to reflect the integrity of S1 maintenance. That is, when integrity of the maintained memory trace is high, performance is correct, but when the maintenance trace is degraded, performance suffers. Although they were often provocative, previous studies have been unable to characterize the conditions leading to degradation in maintenance-related activity.

In the present study, we suggest that selection failures may lead to trace degradation by failing to inhibit either endogenous sources of neural noise or interference from neural noise generated by externally presented stimuli. We found that when ventrolateral PFC differentiated congruent and incongruent distractor processing during the delay interval, subsequent task performance was successful. When congruency-related effects were absent within ventrolateral PFC, performance suffered. We suggest that ventrolateral PFC may provide input to brain regions involved in working memory maintenance processes. One putative recipient site for input is the dorsolateral PFC. As with the ventrolateral PFC, delay activity in the dorsolateral PFC was greater for correct than for incorrect trials. In fact, the magnitude of delay activity within dorso- and ventrolateral regions did not significantly differ when activity within each region was collapsed across congruency conditions. Unlike with ventrolateral PFC, however, dorsolateral PFC delay activity was invariant with distractor type. Perhaps the activity level within the dorsolateral PFC is reliant on processing within the ventrolateral $\mathrm{PFC}$ that is, selection failures within ventrolateral PFC may result in maintenance failures within dorsolateral PFC. These maintenance failures may result from degradation introduced by noise sources that were not appropriately filtered out by ventrolateral PFC. Although our results suggest that dorsolateral PFC may subserve maintenance and receive input from ventrolateral $\mathrm{PFC}$, it is beyond the scope of the present article to determine all potential sites subserving maintenance. Nonetheless, our results do suggest that regions supporting maintenance are neuroanatomically distinct from ventrolateral PFC regions involved in selection and interference resolution.

Recently, Bunge and colleagues (Bunge et al., 2001) investigated the degree of overlap in brain regions sup- 
porting maintenance and proactive interference resolution. They reported that activity within left ventrolateral PFC was significantly correlated with the ability to resolve proactive interference but not the ability to maintain information in working memory. Others, however, have reported positive load-related modulations within dorso- and ventrolateral PFC and suggest that these regions subserve working memory maintenance processes (D’Esposito, Postle, Ballard, \& Lease, 1999; Leung et al., 2002; Rypma \& D’Esposito, 2000; Rypma et al., 1999).

Another approach to determining the role of delay activity in working memory has been to characterize delay activity's ability to "resist distraction" while supporting maintenance processes (Sakai et al., 2002). Similar to our own study, these studies introduced task-irrelevant stimuli during the delay intervals of delayed-recognition tasks. Unlike our study, though, previous studies did not manipulate the domain of distractors but instead varied the absence or presence of distractors across trials. Some of these studies have reported that prefrontal delay interval activity is invariant during distractor presence or absence (Jiang, Haxby, Martin, Ungerleider, \& Parasuraman, 2000; Miller et al., 1996) but that activity within posterior perceptual regions diminishes during distracted delays (Jiang et al., 2000; Miller, Li, \& Desimone, 1993). In contrast, Postle and colleagues (Postle, Druzgal, \& D'Esposito, 2003) recently reported that prefrontal activity diminishes during presentation of delay-spanning distractors, yet activity within posterior perceptual regions appears to be distractor-resistant. They suggest that posterior perceptual regions, and not PFC, support working memory maintenance processes during distracted delays. Despite inconsistencies in the pattern of results reported, all of these studies interpret distractorinvariant activity as reflecting the neural instantiation of maintenance processes that are protected from distractor interference effects.

An alternative interpretation is that activity during both filled and unfilled delays reflects selection mechanisms that are necessary to segregate task-relevant from task-irrelevant information. In line with this interpretation, recent event-related potential (ERP) results from prefrontal lesion patients report that auditory-evoked ERPs time-locked to the presentation of auditory delayspanning distractors were enhanced in frontal-lesion patients relative to control subjects (Chao \& Knight, 1998; Knight, Staines, Swick, \& Chao, 1999). These results suggest that PFC may be necessary to bias posterior perceptual activity so that perceptual processing of distractors is inhibited.

In the context of the present study, ventrolateral PFC may bias posterior perceptual activity within FFA. Specifically, ventrolateral PFC selection mechanisms may result in greater top-down inhibition of face processing during working memory for faces rather than shoes. We observed the opposite pattern, however; face-evoked activity was greater during face than during shoe working memory.
Unlike ERPs, which index neural activity directly, the BOLD technique used in the current fMRI study relies on indirect measures of neural activity via hemodynamic indices. In a recent ERP pilot study (Wencil et al., 2003), we investigated the amplitude of the face-sensitive N170 component evoked by face distractors in a paradigm similar to that used here. N170 responses to face distractors were diminished during face relative to shoe working memory. Although these pilot results are consistent with the view that task-irrelevant distractors may have been perceptually inhibited, it is important to note that the FFA is not the proposed generator site for the N170, and caution should be used in directly relating our fMRI and ERP findings.

Since the hemodynamic requirements for inhibition and excitation may be comparable, it is possible that the pattern of fMRI activity observed in FFA in the present study corresponds to greater inhibition of neural activity during face than doing shoe working memory. Alternatively, greater activity during face versus shoe working memory within the FFA could reflect maintenance of task-relevant faces as well as inhibition of task-irrelevant distractors. Thus, the present results do not allow us to conclusively determine the functional significance of FFA activity during distracted delays. Nonetheless, since FFA congruency effects were present for correct but not for incorrect trials, our results suggest that differential face processing may serve to ensure successful working memory performance.

Finally, most previous studies reporting selectionrelated activity within ventrolateral PFC have used verbally codable stimuli and have found greater activity within left rather than right ventrolateral PFC (Bunge et al., 2001; D'Esposito, Postle, Jonides, \& Smith, 1999; Jonides et al., 1998; Nelson et al., 2003; ThompsonSchill et al., 2002). In the present study, we observed robust left-lateralized activity within these regions both when stimuli were faces and when they were shoes. In sum, the present results support the proposal that left ventrolateral PFC may subserve domain-general representational selection processes to resolve delay-spanning distractor interference.

\section{REFERENCES}

Awh, E., Jonides, J., \& Reuter-Lorenz, P. A. (1998). Rehearsal in spatial working memory. Journal of Experimental Psychology: Human Perception \& Performance, 24, 780-790.

Awh, E., Jonides, J., Smith, E. E., Buxton, R. B., Frank, L. R., Love, T., Wong, E. C., \& GmeindL, L. (1999). Rehearsal in spatial working memory: Evidence from neuroimaging. Psychological Science, 10, 433-437.

BARNes, L., Nelson, J., \& Reuter-Lorenz, P. (2001). Object-based attention and object working memory: Overlapping processes revealed by selective interference effects in humans. Progress in Brain Research, 134, 471-481.

Bunge, S. A., Ochsner, K. N., Desmond, J. E., Glover, G. H., \& Gabrieli, J. D. E. (2001). Prefrontal regions involved in keeping information in and out of mind. Brain, 124, 2074-2086.

Cavada, C., \& Goldman-Rakic, P. S. (1989). Posterior parietal cortex in rhesus monkey: II. Evidence for segregated corticocortical net- 
works linking sensory and limbic areas with the frontal lobe. Journal of Cognitive Neuroscience, 287, 422-445.

Chafee, M. V., \& Goldman-RAKIC, P. S. (1998). Matching patterns of activity in primate prefrontal area $8 \mathrm{a}$ and parietal area 7ip neurons during a spatial working memory task. Journal of Neurophysiology, 79, 2919-2940.

CHAFEe, M. V., \& Goldman-RaKic, P. S. (2000). Inactivation of parietal and prefrontal cortex reveals interdependence of neural activity during memory-guided saccades. Journal of Neurophysiology, $\mathbf{8 3}$ 1550-1566.

CHAO, L. L., \& KNight, R. T. (1995). Human prefrontal lesions increase distractibility to irrelevant sensory inputs. NeuroReport, $\mathbf{6}$, $1605-1610$

Chao, L. L., \& Knight, R. T. (1998). Contribution of human prefrontal cortex to delay performance. Journal of Cognitive Neuroscience, $\mathbf{1 0}$ 167-177.

Constantinidis, C., \& Steinmetz, M. (1996). Neural activity in posterior parietal area 7 a during the delay periods of a spatial memory task. Journal of Neurophysiology, 76, 1352-1355.

CorbetTa, M., Kincade, J. M., \& ShULman, G. L. (2002). Neural systems for visual orienting and their relationships to spatial working memory. Journal of Cognitive Neuroscience, 14, 508-523.

Desimone, R., Chelazzi, L., Miller, E. K., \& Duncan, J. (1995). Neuronal mechanisms of visual attention. In T. V. Papathomas (Ed.), Early vision and beyond (pp. 219-226). Cambridge, MA: MIT Press, Bradford Books.

Desimone, R., \& Duncan, J. (1995). Neural mechanisms of selective visual attention. Annual Review of Neuroscience, 18, 193-222.

D’Esposito, M., Postle, B. R., Ballard, D., \& Lease, J. (1999). Maintenance versus manipulation of information held in working memory: An event-related fMRI study. Brain \& Cognition, 41, 6686.

D’Esposito, M., Postle, B. R., Jonides, J., \& Smith, E. E. (1999). The neural substrate and temporal dynamics of interference effects in working memory as revealed by event-related functional MRI. Proceedings of the National Academy of Sciences, 96, 7514-7519.

EPSTEIN, R., \& KANWISHER, N. (1998). A cortical representation of the local visual environment. Nature, 392, 598-601.

Friston, K. J., Ashburner, J., Frith, C. D., Poline, J.-B., Heather, J. D., \& Frackowiak, R. S. J. (1995). Spatial registration and normalization of images. Human Brain Mapping, 2, 165-189.

Funahashi, S., Bruce, C. J., \& Goldman-Rakic, P. S. (1989). Mnemonic coding of visual space in the monkey's dorsolateral prefrontal cortex. Journal of Neurophysiology, 61, 331-349.

FUSTER, J. M. (1973). Unit activity in prefrontal cortex during delayedresponse performance: Neuronal correlates of transient memory. Journal of Neurophysiology, 36, 61-78.

FUSTER, J. M. (1995). Memory in the cerebral cortex: An empirical approach to neural networks in the human and nonhuman primate. Cambridge, MA: MIT Press.

Fuster, J. M. (1997). The prefrontal cortex: Anatomy, physiology, and neuropsychology of the frontal lobe (3rd ed.). Philadelphia: LippincottRaven.

Giesbrecht, B., \& Mangun, G. R. (2002). The neural mechanisms of top-down control. In H.-O. Karnath, D. Milner, \& G. Vallar (Eds.), The cognitive and neural bases of spatial neglect (pp. 243-257). Oxford: Oxford University Press.

Goggin, J., \& Wickens, D. D. (1971). Proactive interference and language change in short-term memory. Journal of Verbal Learning \& Verbal Behavior, 10, 453-458.

Goldman-RAKIC, P. S. (1987). Circuitry of primate prefrontal cortex and regulation of behavior by representational memory. In J. M. Brookhart \& V. B. Mountcastle (Series Eds.) \& F. Plum (Vol. Ed.), Handbook of physiology: Sec. 1. The nervous system: Vol. 5. Higher functions of the brain (pp. 373-417). Bethesda, MD: American Physiological Society.

Hawkins, H. L., Pardo, V. J., \& Cox, R. D. (1972). Proactive interference in short-term recognition: Trace interaction or competition? Journal of Experimental Psychology, 92, 43-48.

Hopfinger, J. B., Buonocore, M. H., \& Mangun, G. R. (2000). The neural mechanisms of top-down attentional control. Nature Neuroscience, 3, 284-291.

JнA, A. P. ( 2002$)$. Tracking the time-course of attentional involvement in spatial working memory: An event-related potential investigation. Cognitive Brain Research, 15, 61-69.

JHA, A. P., \& MCCARTHY, G. (2000). The influence of memory load upon delay-interval activity in a working-memory task: An eventrelated functional MRI study. Journal of Cognitive Neuroscience, $\mathbf{1 2}$, 90-105.

Jiang, Y., Haxby, J. V., Martin, A., Ungerleider, L. G., \& ParaSURAMAN, R. (2000). Complementary neural mechanisms for tracking items in human working memory. Science, 287, 643-646.

Jonides, J., Smith, E. E., Marshuetz, C., Koeppe, R. A., \& ReuterLORENZ, P. A. (1998). Inhibition in verbal working memory revealed by brain activation. Proceedings of the National Academy of Sciences, 95, 8410-8413.

KAN, I. P., \& THOMPSON-SCHILL, S. L. (2004). Effect of name agreement on prefrontal activity during overt and covert picture naming. Cognitive, Affective, \& Behavioral Neuroscience, 4, 43-57.

Kanwisher, N., McDermott, J., \& Chun, M. M. (1997). The fusiform face area: A module in human extrastriate cortex specialized for face perception. Journal of Neuroscience, 17, 4302-4311.

Kastner, S., Pinsk, M. A., De Weerd, P., Desimone, R., \& UngerLEIDER, L. G. (1999). Increased activity in human visual cortex during directed attention in the absence of visual stimulation. Neuron, 22, 751-761.

KIMBERG, D. Y., \& FARAH, M. J. (1993). A unified account of cognitive impairments following frontal lobe damage: The role of working memory in complex, organized behavior. Journal of Experimental Psychology: General, 122, 411-428.

Knight, R. T., Hillyard, S. A., Woods, D. L., \& Neville, H. J. (1981). The effects of frontal cortex lesions on event-related potentials during auditory selective attention. Electroencephalography \& Clinical Neurophysiology, 52, 571-582.

KNight, R. T., Staines, W. R., Swick, D., \& Chao, L. L. (1999). Prefrontal cortex regulates inhibition and excitation in distributed neural networks. Acta Psychologica, 101, 159-178.

LaBar, K. S., Gitelman, D. R., Parrish, T. B., \& Mesulam, M.-M. (1999). Neuroanatomic overlap of working memory and spatial attention networks: A functional MRI comparison within subjects. NeuroImage, 10, 695-704.

LeUnG, H.-C., GORE, J. C., \& Goldman-Rakic, P. S. (2002). Sustained mnemonic response in the human middle frontal gyrus during on-line storage of spatial memoranda. Journal of Cognitive Neuroscience, 14, 659-671.

LURIA, A. R. (1973). The working brain: An introduction to neuropsychology (B. Haigh, Trans.). Harmondsworth, U.K.: Penguin.

LuRIA, A. R., \& HomsKaYA, E. D. (1970). Frontal lobes and the regulation of arousal processes. In D. I. Mostofsky (Ed.), Attention: Contemporary theory and analysis (pp. 58-72). New York: AppletonCentury-Crofts.

MALMO, R. (1942). Interference factors in delayed response in monkeys after removal of frontal lobes. Journal of Neurophysiology, 5, 295-308.

Mangun, G. R., Buonocore, M. H., Girelli, M., \& JHA, A. P. (1998). ERP and fMRI measures of visual spatial selective attention. Human Brain Mapping, 6, 383-389.

MARCER, D. (1972). Interference and short-term retention. British Journal of Psychology, 63, 533-536.

May, C. P., HASHER, L., \& KANE, M. J. (1999). The role of interference in memory span. Memory \& Cognition, 27, 759-767.

McCarthy, G., Puce, A., Gore, J. C., \& Allison, T. (1997). Facespecific processing in the human fusiform gyrus. Journal of Cognitive Neuroscience, 9, 605-610.

MCInTYRe, J. S., StoJAK, R. A., \& Mostoway, W. (1973). Individual organization and release from proactive interference. Journal of Experimental Psychology, 98, 164-168.

Milham, M. P., Banich, M. T., Webb, A., Barad, V., Cohen, N. J., WszaleK, T., \& Kramer, A. F. (2001). The relative involvement of anterior cingulate and prefrontal cortex in attentional control depends on nature of conflict. Cognitive Brain Research, 12, 467-473. 
Miller, E. K., \& CoHEN, J. D. (2001). An integrative theory of prefrontal cortex function. Annual Review of Neuroscience, 24, 167-202.

Miller, E. K., Erickson, C. A., \& Desimone, R. (1996). Neural mechanisms of visual working memory in prefrontal cortex of the macaque. Journal of Neuroscience, 16, 5154-5167.

Miller, E. K., LI, L., \& DeSIMONE, R. (1991). A neural mechanism for working and recognition memory in inferior temporal cortex. Science, 254, 1377-1379.

Miller, E. K., LI, L., \& Desimone, R. (1993). Activity of neurons in anterior inferior temporal cortex during a short-term memory task. Journal of Neuroscience, 13, 1460-1478.

Nelson, J. K., Reuter-Lorenz, P. A., Sylvester, C.-Y. C., Jonides, J., \& Sмith, E. E. (2003). Dissociable neural mechanisms underlying response-based and familiarity-based conflict in working memory. Proceedings of the National Academy of Sciences, 100, 11171-11175.

PessoA, L., Kastner, S., \& UngerleIder, L. G. (2003). Neuroimaging studies of attention: From modulation of sensory processing to topdown control. Journal of Neuroscience, 23, 3990-3998.

Postle, B. R., Druzgal, T. J., \& D'Esposito, M. (2003). Seeking the neural substrates of working memory storage. Cortex, 39, 927-946.

Postle, B. R., Zarahn, E., \& D'Esposito, M. (2000). Using event-related fMRI to assess delay-period activity during performance of spatial and nonspatial working memory tasks. Brain Research Protocols, 5, 57-66.

Ranganath, C., DeGutis, J., \& D’Esposito, M. (2004). Categoryspecific modulation of inferior temporal activity during working memory encoding and maintenance. Cognitive Brain Research, 20, 37-45.

RaO, S. G., Williams, G. V., \& GoldMan-RaKic, P. S. (1999). Isodirectional tuning of adjacent interneurons and pyramidal cells during working memory: Evidence for microcolumnar organization in PFC. Journal of Neurophysiology, 81, 1903-1916.

RYPMA, B., \& D'EsPosito, M. (2000). Isolating the neural mechanisms of age-related changes in human working memory. Nature Neuroscience, 3, 509-515.

RYPma, B., Prabhakaran, V., Desmond, J. E., Glover, G. H., \& GABRIELI, J. D. E. (1999). Load-dependent roles of frontal brain regions in the maintenance of working memory. Neurolmage, 9, 216-226.
SAKaI, K., Rowe, J. B., \& Passingham, R. E. (2002). Active maintenance in prefrontal area 46 creates distractor-resistant memory. $\mathrm{Na}$ ture Neuroscience, 5, 479-484.

THOMPSON-SCHILL, S. L. (2003). Neuroimaging studies of semantic memory: Inferring "how" from "where." Neuropsychologia, 41, 280292.

Thompson-Schill, S. L., D’Esposito, M., Aguirre, G. K., \& Farah, M. J. (1997). Role of left inferior prefrontal cortex in retrieval of semantic knowledge: A reevaluation. Proceedings of the National Academy of Sciences, 94, 14792-14797.

ThOMPSON-Schill, S. L., Jonides, J., Marshuetz, C., Smith, E. E., D'Esposito, M., Kan, I. P., KNight, R. T., \& Swick, D. (2002). Effects of frontal lobe damage on interference effects in working memory. Cognitive, Affective, \& Behavioral Neuroscience, 2, 109-120.

TippetT, L. J., Gendall, A., Farah, M. J., \& Thompson-Schill, S. L. (2004). Selection ability in Alzheimer's disease: Investigation of a component of semantic processing. Neuropsychology, 18, 163-173.

Wencil, E. B., Rauch, S., Scheinker, D., \& Jha, A. P. ( $\overline{20} 03)$. Faces are not always special! Working memory studies of distractor intrusiveness [Abstract]. Cognitive Neuroscience Society Abstracts: 2003 Annual Meeting, F236.

Wilson, F. A. W., Ó Scalaidhe, S. P., \& Goldman-RaKic, P. S. (1993). Dissociation of object and spatial processing domains in primate prefrontal cortex. Science, 260, 1955-1958.

Zarahn, E., Aguirre, G. [K.], \& D’Esposito, M. (1997a). A trialbased experimental design for fMRI. NeuroImage, 6, 122-138.

Zarahn, E., Aguirre, G. K., \& D'Esposito, M. (1997b). Empirical analyses of BOLD fMRI statistics. I. Spatially unsmoothed data collected under null-hypothesis conditions. NeuroImage, 5, 179-197.

Zarahn, E., Aguirre, G. K., \& D'Esposito, M. (1999). Temporal isolation of the neural correlates of spatial mnemonic processing with fMRI. Cognitive Brain Research, 7, 255-268.

(Manuscript received December 8, 2004; revision accepted for publication December 20, 2004.) 